\title{
Using Rummble as a Personalised Travel Guide
}

\author{
Clive J. Cox
}

\begin{abstract}
Rummble Ltd (www.rummble.com) is an online mobile local content provider that gives users access to trusted spatially-located content via the web and mobile. Rummble brands itself as a "Personalised travel guide written by your friends". Users sign up and build a social network. They upload reviews of places they have visited and provide ratings for them. The service carries out a trust network analysis on each person's friends network and determines the individually most trusted people in their network. The service can then determine the likelihood a user will enjoy visiting a particular place by combining the trust (and ratings) for each person who has reviewed the place in question. For example, a user visiting the Barri-Gotic in Barcelona can immediately find via their mobile phone bars and restaurants they might like which have been reviewed by trusted people from their friends network and rated highly.
\end{abstract}

\section{Demonstration Overview}

The demonstration will show both the web and mobile interfaces provided by the service. The web service provides the usual selection of social networking tools: mail, blogs, photo upload, message board. There is functionality related to the mobile domain, SMS sending, control of who can send the user SMSs and when. Also, there is functionality related to spatial content: the user can specify their future trips and find out who will be in the same location and they can say where and when they will be periodically each day (e.g., office, home).

The core of the web service is the Rummble Explorer which allows users to explore via a Google maps mashup locations and find content that has been entered by members of their social network. This content can be filtered by tags and distance inside the social network and most pertinently by the trust the user has in the

Clive J. Cox

Rummble.com, Cambridge, England e-mail: clive.cox@rummble.com

Please use the following format when citing this chapter:

Cox, C. J., 2008, in IFIP International Federation for Information Processing, Volume 263; Trust Management II; Yücel Karabulut, John Mitchell, Peter Herrmann, Christian Damsgaard Jensen; (Boston: Springer), pp. 359-362. 
content. Fig.1 shows a view of Barcelona with one "Rummble" for Cafe Schilling highlighted, showing its trust.
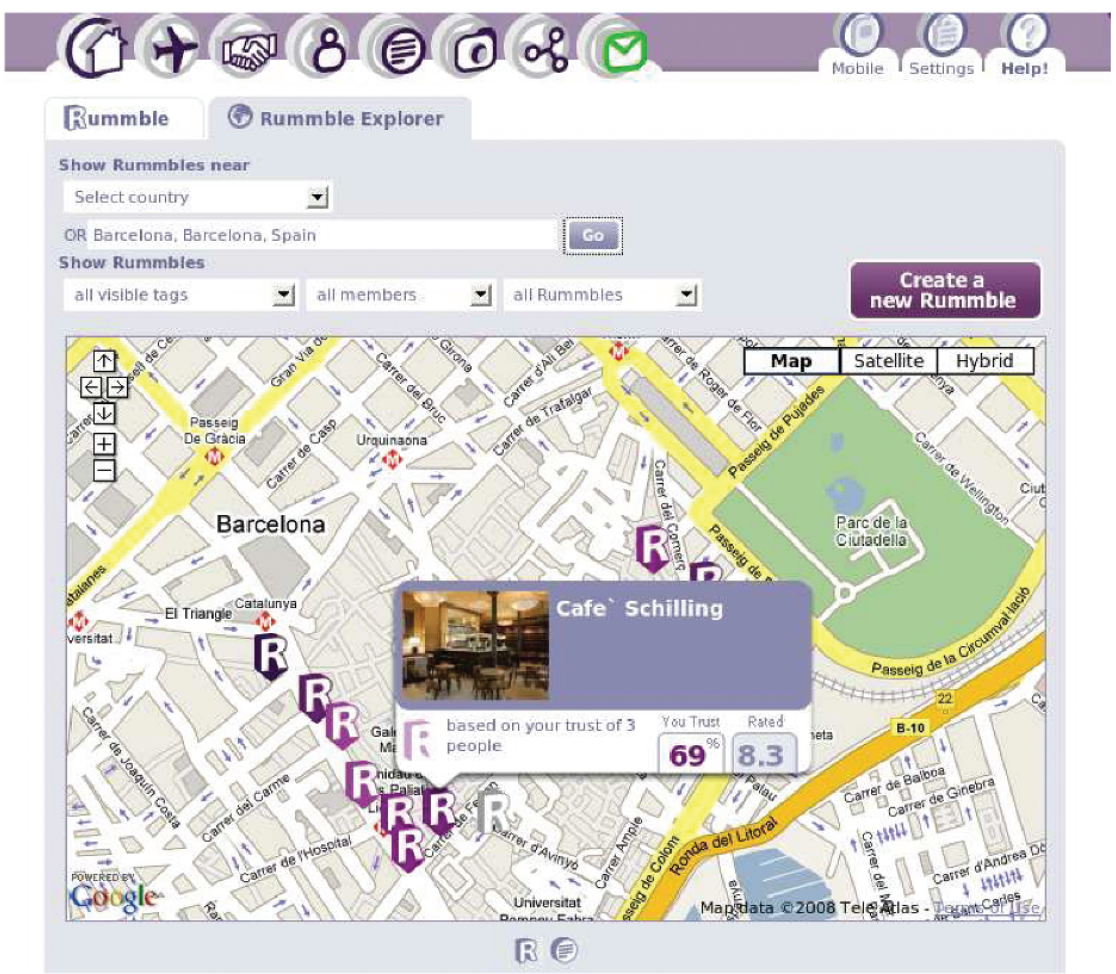

Fig. 1 Trust for a Cafe in Barcelona

The trust for the content is derived from the user's trust of the three people who reviewed Cafe Schilling and the rating is an expected rating derived from this trust and the ratings given by the three users showing what the system's present guess is for what the user's rating would be if they visited the cafe and rated it.

The user can then drill down into the actual review for Cafe Schilling and read the individual reviews and ratings and see the indivudal trust they have for each reviewer, as shown in Fig.2.

In order to lessen the possibility of information overload the Rummble Explorer can show only the content that the service believes the user might enjoy. This ability which is of even greater importance in mobile environments is available on the mobile Java application that Rummble provides. The demonstration will show this application in use. The mobile application is a rich client which provides functionality to:

1. Set your location via a map, GPS, or place name anywhere in the world.

2. Browse via a map the Rummble content and upload new content. 


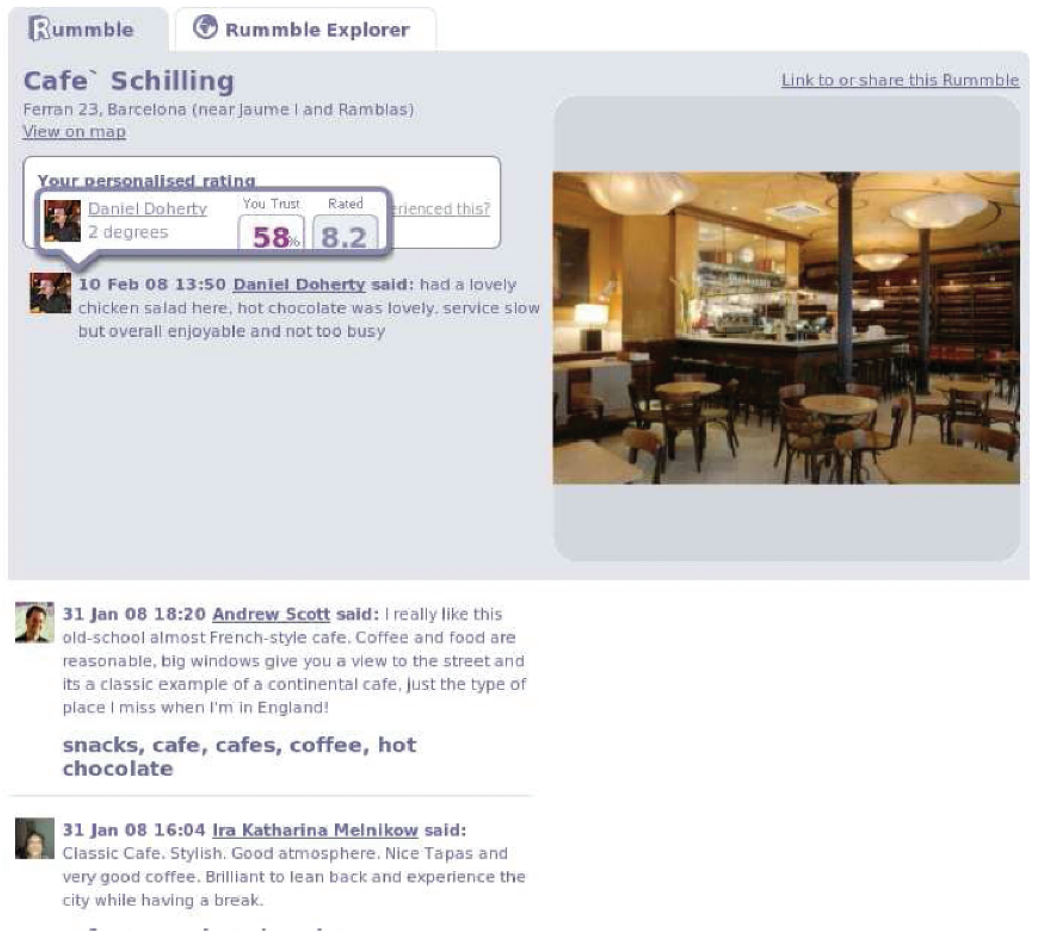

Fig. 2 Reviews for Cafe Schilling

3. Chat to Rummble users and on external IM networks (e.g., Yahoo, MSN, IRC etc.)

Fig. 3 shows the mobile application showing the places it recommends for the part of Barcelona displayed on the map. As with the web application the user can read the indivudal reviews for the place in question and see their trust for each reviewer. By utilizing filtering based on the trust network analysis the user can be provided with a smaller and hopefully more appropriate selection of content which will allow them to quickly find places of interest while on the move with their mobile.

\section{Conclusion}

The recommendations provided by Rummble are based on private and transitive trust between users, and thereby provide a highly personalised service. Rummble harnesses social networks together with the Internet and mobile networks to assist 


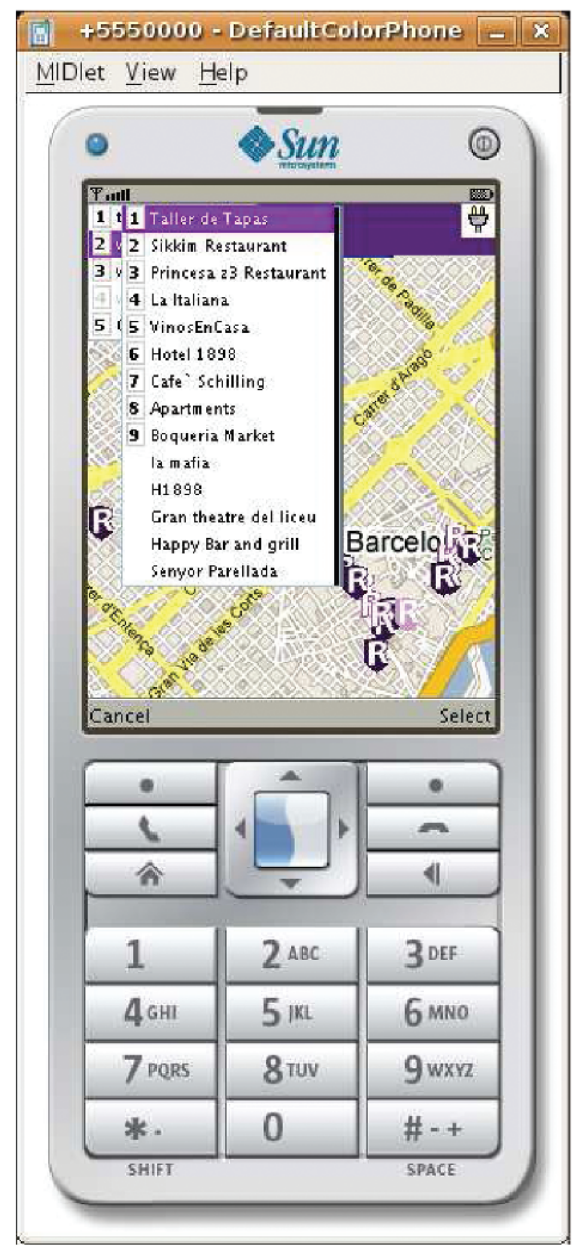

Fig. 3 Recommendations on the mobile application

people in finding places and selecting services that they will find interesting, useful and enjoyable. 